\title{
The Role of Mindfulness and Life Satisfaction in Psychological Distress During the COVID-19 Lockdown in New Zealand: a Quasi-experimental Study
}

\author{
Jayne M. Hartstone ${ }^{1} \cdot$ Oleg N. Medvedev $^{1}$
}

Accepted: 16 August 2021 / Published online: 26 August 2021

(c) The Author(s), under exclusive licence to Springer Science+Business Media, LLC, part of Springer Nature 2021

\begin{abstract}
Objectives Quantitative research on the psychological effects of lockdown conditions during the COVID-19 pandemic is needed to inform mental health interventions which aim to alleviate potential adverse effects. The goal of this study was to investigate psychological distress during the lockdown in New Zealand.

Methods We implemented a longitudinal quasi-experimental research design using a sample $(n=81)$ who completed surveys on mindfulness, satisfaction with life, and mental health indicators at three time-points, separated by at least 2-week intervals. The sample was divided into two parts, the baseline group $(n=44)$ and the lockdown group $(n=37)$. The baseline group completed the surveys the first time prior to lockdown, and mostly completed the second and third surveys during lockdown. The lockdown group mostly completed the survey for the first time during lockdown and the second and third surveys during or after lockdown.

Results Mindfulness and satisfaction with life at baseline significantly predicted lower levels of depression, anxiety, and stress during uncertain and emergency conditions before and during lockdown. The baseline group experienced significantly less anxiety and stress during lockdown compared to just prior to the lockdown (baseline condition).

Conclusions Individuals who have higher levels of mindfulness and those with greater life satisfaction experience significantly less depression, anxiety, and stress over time, during both uncertain and emergency conditions such as during the threat of COVID-19. The finding of anxiety and stress reduction during lockdown may be specific to New Zealand, as conditions differ in many ways from those in other countries. Preventative measures which increase mindfulness and satisfaction with life could help alleviate depression, anxiety, and stress during uncertain and emergency conditions.
\end{abstract}

Keywords COVID-19 $\cdot$ Lockdown $\cdot$ Depression $\cdot$ Anxiety $\cdot$ Stress $\cdot$ Mindfulness $\cdot$ Satisfaction with life

The coronavirus pandemic has caused an alarming loss of human life and continued danger, resulting in over 4.3 million deaths and more than 207 million cases worldwide (Worldometer, 2021). The International Labour Organization (2021) reported that the livelihoods of millions of people have been seriously disrupted due to the effects of the pandemic, and projections indicated that the number of people experiencing poverty could increase by 119 million-124 million in 2021 (Beaumont, 2021). The effects of COVID19 have also led to widespread and severe food insecurity

Oleg N. Medvedev

oleg.medvedev@waikato.ac.nz

1 School of Psychology, University of Waikato, Rm K 1.23, Hillcrest, Private Bag 3105, Hamilton 3240, New Zealand globally, which disproportionately disadvantages those in low- and middle-income countries (The World Bank, 2021).

Bereavement, fear of infection, isolation, and financial insecurity due to the effects of the pandemic are precipitating mental health problems and exacerbating existing conditions (World Health Organization, 2020). Many individuals may be experiencing elevated levels of anxiety as well as increased insomnia and alcohol and/or drug intake. Such factors have taken an enormous toll, with mental health care facilities in $93 \%$ of nations around the world disrupted or halted, according to a recent survey by the World Health Organization (2020).

A recent study from the USA (Ettman et al., 2020) with a large community sample of adult participants demonstrated that the prevalence of depression in March and April 2020 increased by more than $300 \%$ from levels prior to the 
pandemic. The authors concluded that the pandemic signified a traumatic event in the lives of many Americans, resulting in high levels of psychological distress. Similarly, a study by Twenge and Joiner (2020) featuring a nationally representative sample showed that American adults were approximately three times more likely to report anxiety symptoms during the pandemic (April and May 2020) than they were in pre-COVID-19 times. However, results from this study showed that anxiety levels decreased slightly between April and May 2020, whereas levels of depressive symptoms continued to rise.

A study facilitated in the UK (Shevlin et al., 2020), also with a large community sample of adult participants, demonstrated an increase of depressive and anxiety symptoms following the onset of the pandemic. In the study, the prevalence of depression during the pandemic was reported to be $22 \%$, which is higher than pre-COVID-19 times, although the increase is not substantial. However, the data during the pandemic were collected in March 2020, which was relatively early in the history of the virus. Due to the spike in number of cases and resulting lockdowns in the UK (McMullan et al., 2021), the prevalence of depression and anxiety may have increase further.

The results of a longitudinal study conducted in China (Wang et al., 2020) with a very large community sample made up of participants of various ages (children, adolescents, and adults) demonstrated that despite a sharp increase in the number of COVID-19 cases between the first and second surveys, there was no significant change in depression or anxiety levels. The first surveys were completed from January 31 to February 2, 2020, and the second surveys were open from February 28 until March 1, 2020. The findings are therefore inconsistent with those of the aforementioned studies from the USA (Ettman et al., 2020) and the UK (Shevlin et al., 2020); however, it is important to note that both surveys in the study by Wang et al. (2020) were conducted during the pandemic (unlike the American and UK studies, in which pre-COVID-19 levels of distress variables were measured). Wang et al. (2020) posited that the swift, responsive action taken by the Chinese Government reduced the spread of the virus and such measures could be generally protective against the development of mental health conditions.

While there is no substitute for physical safety, financial security, and adequate food supplies, research is nevertheless critical in determining the psychological protective factors against adverse mental health outcomes during such challenging times. Research has shown that various aspects of dispositional mindfulness are predictive of lower levels of psychological distress among general and student populations (Medvedev et al., 2018a). The results also demonstrated that nonjudgment was the strongest predictor of lower levels of depression, anxiety, and stress for both groups, with a moderate effect size. Another study by Medvedev et al. (2020) using network analysis with a sample made up of equal numbers of students and members of the general public showed that all mindfulness facets interact with one another to achieve health benefits, while having a nonjudgmental attitude specifically was highly and inversely linked to depression, negative affect, and anxiety. The results of this study further indicated that acting with awareness and non-reactivity were inversely linked with anxiety and stress, respectively.

Research has also demonstrated that mindfulness-based interventions help significantly decrease the symptoms of depression (Strauss et al., 2014) and lower stress (Chiesa $\&$ Serretti, 2009). Similarly, mindfulness- and acceptancebased interventions have been demonstrated to reduce anxiety symptoms (Vøllestad et al., 2012). Satisfaction with life has been shown to have significant, moderate inverse correlations with distress variables (Headey et al., 1993). It is therefore instructive to investigate the potentially protective effects of mindfulness and satisfaction with life during the COVID-19 pandemic.

Mindfulness has been defined as a non-judgmental and non-reactive awareness of, and attention to the present moment, approached with as much openheartedness as possible (Kabat-Zinn, 2015). It encompasses the nonjudgmental observation of both internal and external phenomena as they occur (Baer, 2003). Operating on autopilot or paying little attention to one's own actions is therefore the opposite to mindfulness (Nagy \& Baer, 2017). Rumination, preoccupation with the past, and worry or anxiety pertaining to the future hamper attention to the present moment and thereby thwart mindfulness (Brown \& Ryan, 2003). Conversely, the nonjudgmental observation of emotions, cognitions, and experiences which are key to mindfulness allow for increased self-regulation and selection of adaptive responses, rather than impulsive or automatic reactions (Roemer et al., 2021b).

Conceptions of mindfulness emanated from ancient Eastern traditions but have more recently been adopted in Western settings (Nagy \& Baer, 2017). Mindfulness is conceived as an intrinsic quality of the mind; however, it can be further refined through practices such as meditation (Kabat-Zinn, 2015). Mindfulness which has been cultivated intentionally often leads to spontaneous manifestations of effortless mindfulness.

The capacity of mindfulness and mindfulness-based interventions to reduce psychological distress with a wide range of samples has been well documented (Krägeloh et al., 2019). For example, the results of a study with a sample of university students studying in Spain (Gallego et al., 2014) demonstrated that depression, anxiety, and stress levels were significantly lowered post-intervention among a group who received mindfulness-based cognitive therapy compared to 
a control group. In a study by Joss et al. (2019), a sample of adults living in the USA who had been maltreated during childhood were placed into either a mindfulness-based behavioral intervention group or a waitlist control group. The results indicated that the intervention group reported significantly greater increases in mindfulness and reductions of anxiety and stress than the waitlist control group. Similarly, the results of another study (Roemer et al., 2021b) showed distress among a sample of unemployed young adults in New Zealand was significantly reduced following participation in a low-dose mindfulness-based intervention, and those with higher levels of dispositional mindfulness and well-being benefited more from the intervention.

Life satisfaction is defined as the "the extent to which a person finds life rich, meaningful, full, or of high quality" by the American Psychological Association (2020, para. 1). Satisfaction with life may be defined as the level of similarity between desired conditions and one's individual perception of reality (Cummins and Nistico 2002); therefore, it is a cognitive process, as described by Diener et al. (1985). The degree to which the individual is satisfied with life usually depends on an assessment of how well one's needs have been met in the past as well as an estimation of how likely they are to be satisfied in the future (Veenhoven, 2017).

Interventions which help increase satisfaction with life have been shown to have advantageous effects on mental health outcomes. For example, a recent study by Koydemir and Sun-Selış1k (2016) demonstrated that following a strengths-based program, student participants from a university in Turkey who were placed in an intervention group reported a significant increase in life satisfaction as well as psychological well-being compared to a control group. Adult participants living in Brazil who completed a gratitude-writing intervention in a recent clinical trial (Cunha et al., 2019) reported a significant increase in life satisfaction and significant decreases of depressive symptoms over time. In another study from Hong Kong (Auyeung \& Mo, 2019), university student participants who completed an online version of the Best Possible Self writing intervention reported significant gains of flourishing (conceptually similar to satisfaction with life in this context) and significantly reduced levels of depression compared to a control group.

Baseline levels of mindfulness and satisfaction with life may affect levels of distress resulting from the impact of the COVID-19 pandemic, and more specifically, lockdown conditions. Levels of mindfulness and satisfaction with life must therefore be controlled at baseline. However, this has rarely been implemented in past research with a few notable exceptions such as the study by Roemer et al. (2021b).

Research on the psychological effects caused by lockdown must be specific to each country as lockdown conditions vary substantially from country to country. Indeed, the lockdown conditions in New Zealand were unique in many ways and were described as among some of the strictest in the world by New Zealand's Prime Minister, Jacinda Ardern (Jones, 2020). All new arrivals into New Zealand were required to go into a period of self-isolation from March 16, 2020, except for people travelling from some Pacific nations, which were largely unaffected by coronavirus at the time. There were only 102 cases of coronavirus and no deaths when New Zealand entered its first lockdown.

Unlike other countries such as the UK, New Zealand closed its borders to almost all non-citizens and non-residents, which dramatically decreased the number of people who had the virus entering the country (Jones, 2020). By the end of April, New Zealand had tested members of the public for coronavirus at a rate of 2190 per 100,000 people, which was a stark contrast to the USA where 1140 per 100,000 people had been tested despite many more live cases (Gunia, 2020; The Atlantic Monthly Group, 2021). The personal requirements of lockdown in New Zealand were also significantly different to those of other nations. For example, individuals residing in New Zealand were allowed to go out in public to exercise during lockdown (Nielson, 2020), while in Russia, citizens were not permitted to do so (Coronavirus: Lockdown eased in Moscow after nine weeks, 2020). Furthermore, existing literature shows that levels of depression and anxiety in the USA (Ettman et al., 2020) and UK (Shevlin et al., 2020) during the pandemic increased; however, there was no significant rise of these conditions in China (Wang et al., 2020). Longitudinal research from different countries around the world is needed to investigate the impact of the pandemic and lockdown conditions on psychological distress.

Evidence from both intervention and cross-sectional studies carried out before the coronavirus pandemic demonstrated that dispositional mindfulness and satisfaction with life may act as protective factors against psychological distress. Therefore, the first aim of the current study was to explore whether baseline levels of mindfulness and satisfaction with life were inversely predictive of psychological distress during the coronavirus pandemic. Based on the findings of previous studies, it was hypothesized that individuals with higher levels of mindfulness and satisfaction with life would experience lower levels of depression, anxiety, and stress over time. As very little research regarding the effects of the lockdown on depression, anxiety, and stress had been carried out with New Zealand samples, a further aim of this study was to investigate such effects using a quasi-experimental design. One of the benefits of using quasi-experimental designs is that they have high generalizability to current life contexts due to their ecological validity. Given the previous research, it was hypothesized that levels of depression, anxiety, and stress would increase between the baseline and 
lockdown conditions. We also explored the effects of individual mindfulness facets on the distress variables over time.

\section{Method}

\section{Participants}

The participants $(n=81)$ were students studying psychology at under-graduate level at the University of Waikato, all of whom were living in New Zealand during the study. The participants' ages ranged from 18 to 50 years, with a mean age of 24.31 years $(S D=7.82)$. There were over four times as many female participants (83\%) than there were male participants (17\%). The sample comprised NZ European (62\%), Māori (indigenous peoples of New Zealand) (17\%), Asian (15\%), and other ethnicities (6\%). There were no significant demographic differences between the baseline group and lockdown group (see Table 1).

A part of the sample, the baseline group, completed the surveys the first time between March 16, 2020, and

Table 1 Demographic characteristics of the baseline group $(n=44)$ and the lockdown group $(n=37)$

\begin{tabular}{llll}
\hline & $\begin{array}{l}\text { Baseline group } \\
(n=44)\end{array}$ & $\begin{array}{l}\text { Lockdown group } \\
(n=37)\end{array}$ & $\begin{array}{l}\text { Test of } \\
\text { statistical } \\
\text { difference }\end{array}$ \\
\hline $\begin{array}{l}\text { Mean Age }(S D) \\
\text { Sex } n(\%)\end{array}$ & $23.93(7.92)$ & $24.76(7.78)$ & $p>0.05^{\mathrm{a}}$ \\
Females & $34(77.3)$ & $33(89.2)$ & $p>0.05^{\mathrm{b}}$ \\
Ethnicity $n(\%)$ & & & $p>0.05^{\mathrm{b}}$ \\
European & $25(56.8)$ & $25(67.6)$ & \\
Māori & $9(20.5)$ & $5(13.5)$ & \\
Asian & $8(18.2)$ & $4(10.8)$ & \\
Other & $2(4.6)$ & $3(6.1)$ & \\
\hline
\end{tabular}

Note: ${ }^{\mathrm{a}}$-test. ${ }^{\mathrm{b}} \chi^{2}$ test
March 25, 2020, which was prior to the first lockdown in New Zealand (see Fig. 1). Participants in the baseline group mostly completed the second and third surveys during lockdown. The other part of the sample, the lockdown group, mostly completed the survey for the first time between March 26, 2020, and April 27, 2020, which was during lockdown (see Fig. 1). Participants in the lockdown group completed the second and third surveys during or after lockdown.

\section{Procedure}

The Human Research Ethics Committee at the University of Waikato (New Zealand) granted ethical approval for this study prior to data collection. Information on the purposes of the study, how the data would be used, confidentiality, and participation was provided online, prior to the commencement of each survey. One hundred and ninety-eight students studying a psychology research paper at under-graduate level at the University of Waikato, New Zealand, were invited to take part during a brief presentation prior to a lecture on campus. Ninety-three percent of the students in the class participated in the first survey. Forty-six percent of the students in the class completed the first and second surveys, and $41 \%$ of the entire class completed all three surveys (see Fig. 2). Participants gave informed consent online before each questionnaire. The participants' data was anonymized. Student participants received a small amount of course credit for completing each survey.

The three surveys (one for each time point) opened on March 16, 2020, 5 days before the introduction of the alert level system by the New Zealand Government and a week before the public were given $48 \mathrm{~h}$ to prepare for lockdown (Supplementary Table S1). The national lockdown (Alert Level 4) in New Zealand lasted for approximately one month (Strongman, 2020), which is a relatively short

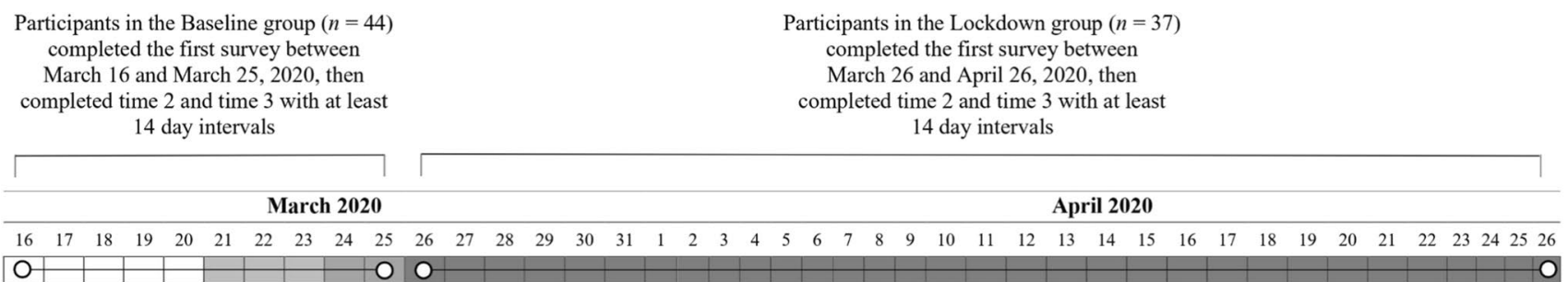

$=$ No alert

$=$ Alert level 2

$=$ Alert level 3

$=$ Alert level 4

Fig. 1 Timeline showing when the baseline group participants $(n=44)$ and the lockdown group participants $(n=37)$ completed the surveys and the alert levels in New Zealand (2020) 
Fig. 2 Flow chart of participant recruitment and attrition, showing how many students participated and how many declined the invitation or left the study at each stage

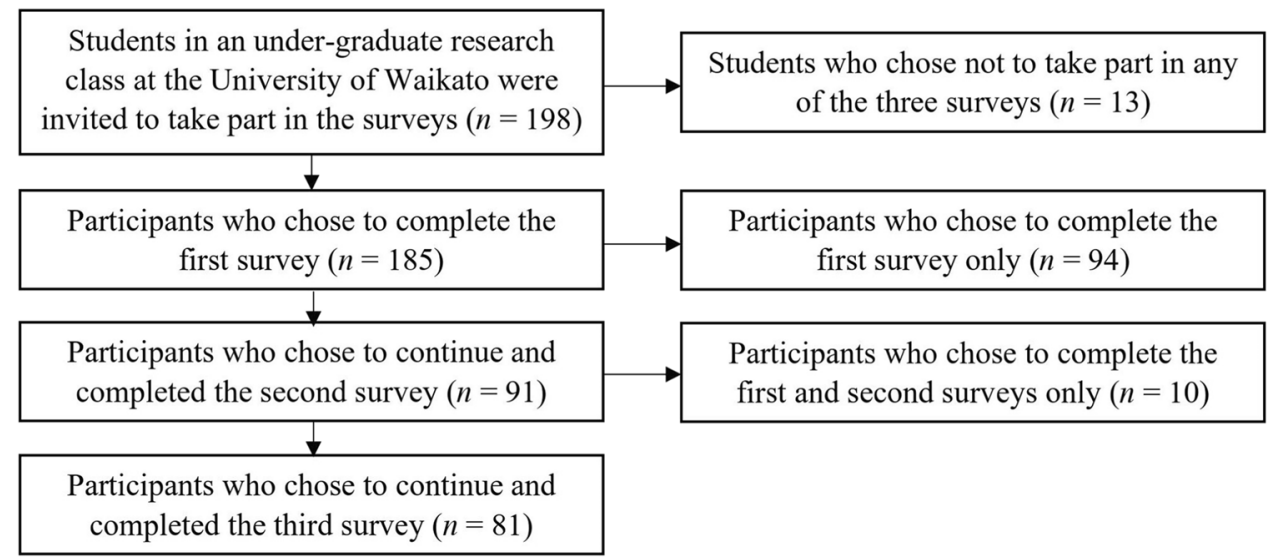

duration compared to many other countries. The surveys remained open until June 19, 2020, at which time all of New Zealand was back in Level 1.

\section{Measures}

In this study, we used the short version of the Five Facet Mindfulness Questionnaire (FFMQ-18), because we wanted to keep our survey short, given it was administered at three separate time points (long surveys may result in higher levels of participant attrition and affect the accuracy of participants' responses). In our main analysis, we used the total mindfulness score because it was found to be more reliable for the short version of the scale compared to the individual facets of mindfulness scores (Medvedev et al., 2018b). Moreover, recent network analysis conducted by Medvedev et al. (2020) demonstrated that all individual mindfulness facets contribute to the overall mindfulness construct in an interactive way, meaning that individual facets may not adequately represent the construct. Therefore, mindfulness was measured using the 18-item Five Facet Mindfulness Questionnaire (FFMQ-18), a self-report form covering five mindfulness domains: act with awareness, describe, nonjudge, non-react, and observe (Medvedev et al., 2018b). The FFMQ-18 uses a 5-point Likert-type scale, with response options from 1 (never or very rarely true) to 5 (very often or always true). The 18 -item version has been shown to have good reliability and is considered a valid measure of trait mindfulness, with Cronbach's alphas ranging from 0.79 to 0.86 when measured over three occasions (Truong et al., 2020). In the current study, Cronbach's alpha ranged from 0.85 to 0.88 over three time points.

The Satisfaction With Life Scale (SWLS) is a widely used 5-item self-report questionnaire which measures the extent to which individuals are satisfied with their lives. The measure requires individuals to rate their level of endorsement for each item by the means of a 6-point Likert-type scale, with response options ranging from strongly agree to strongly disagree. It displays high internal consistency, evidenced by a Cronbach's alpha of 0.87 (Diener et al., 1985). In the current study, Cronbach's alpha ranged from 0.86 to 0.91 over three time points. The SWLS also displays sound test-retest reliability, with a correlation coefficient of 0.82 (Diener et al., 1985). Research has shown convergent and discriminant validities are satisfactory for this measure (van Beuningen, 2012). Well-established psychometric properties and brevity were major considerations for using this scale in the current study.

The Depression, Anxiety and Stress Scales (DASS-21) are an abridged version of the original 42-item self-report measure authored by Lovibond and Lovibond (1993). The measure requires individuals to rate their level of endorsement for each of the 21 items on a 4-point Likert-type scale based on how they felt over the past week, with options ranging from 0 (did not apply to me at all-never) to 3 (applied to me very much, or most of the time-almost always). The DASS-21 contains three 7-item subscales, depression, anxiety, and stress, which each have been shown to demonstrate very high internal consistency, with Cronbach's alphas of 0.94, 0.87, and 0.91, respectively (Antony et al., 1998). In the current study, Cronbach's alpha for the entire scale was 0.92 for all three time points. The concurrent validity ratings of the DASS-21 subscales were moderate to high when scores were compared to those of other frequently used measures of depression and anxiety (Antony et al., 1998). The DASS-21 is a reliable and relatively short scale, which is suitable to assess depression, anxiety, and stress in both clinical and non-clinical populations, providing sound reasons for using this scale in the current study.

The demographic section of the surveys featured three items. The items asked participants to report their sex, age, and ethnicity. Participants were asked to check a box for their sex (male or female) and ethnicity (European/Pākehā, Māori, Pasifika, Asian, or other). The item on age requested participants to input their age in years. 


\section{Data Analyses}

Descriptive statistics were computed using IBM SPSS v27. A multi-level model was used with "time" as a level 1 predictor. The grouping variable (i.e., baseline vs lockdown group) was then entered as a level 2 predictor to investigate the effects of the lockdown on mental health constructs such as anxiety, depression, and stress. Mindfulness and life satisfaction levels at Time 1 were not significantly different between the baseline and lockdown groups, which permitted us to add these variables as covariates of the outcome variables in the mixed model. Post hoc tests were conducted to investigate mean differences both within and between groups where significant main or interaction effects were found. In addition, we explored the overall contribution of individual mindfulness facets to depression, anxiety, and stress after controlling for effects of group and time, using multiple linear regression. We entered anxiety, stress, and depression, at Times 2 and 3 as outcomes and the five mindfulness facets measured at baseline as predictors, while controlling for the respective distress variables at Time 1 and the grouping variable (baseline vs lockdown). For all regression models, depression, anxiety, or stress at Time 1 was entered as a predictor in the first step followed by the group variable (baseline vs lockdown) in the second step, while all mindfulness facets were entered in a stepwise fashion in the next step, which permitted us to extract the most important predictor while simultaneously controlling for variance shared among mindfulness facets (see Medvedev et al., 2018a and Roemer et al., 2021a).
Table 2 Descriptive statistics including skewness and kurtosis values of the baseline group $(n=44)$ and lockdown group $(n=37)$ across the study variables at all time points

\begin{tabular}{|c|c|c|c|c|c|c|}
\hline Variable & Mean & $S D$ & Min & Max & Skewness & Kurtosis \\
\hline \multicolumn{7}{|l|}{ Baseline group } \\
\hline FFMQ-18 Time 1 & 59.45 & 11.76 & 38 & 88 & 0.38 & -0.33 \\
\hline FFMQ-18 Time 2 & 60.64 & 11.22 & 42 & 86 & 0.27 & -0.65 \\
\hline FFMQ-18 Time 3 & 61.20 & 10.85 & 38 & 88 & 0.34 & -0.04 \\
\hline SWLS Time 1 & 20.66 & 5.28 & 5 & 30 & -0.68 & 0.60 \\
\hline SWLS Time 2 & 20.61 & 5.68 & 8 & 30 & -0.37 & -0.77 \\
\hline SWLS Time 3 & 21.41 & 4.79 & 9 & 30 & -0.48 & -0.23 \\
\hline Depression (DASS-21) Time 1 & 4.82 & 5.01 & 0 & 20 & 1.08 & 0.64 \\
\hline Depression (DASS-21) Time 2 & 4.93 & 3.87 & 0 & 13 & 0.54 & -1.01 \\
\hline Depression (DASS-21) Time 3 & 5.50 & 4.57 & 0 & 15 & 0.56 & -1.01 \\
\hline Anxiety (DASS-21) Time 1 & 5.02 & 4.14 & 0 & 15 & 0.92 & 0.25 \\
\hline Anxiety (DASS-21) Time 2 & 3.41 & 3.47 & 0 & 12 & 1.21 & 0.64 \\
\hline Anxiety (DASS-21) Time 3 & 3.30 & 3.12 & 0 & 13 & 1.04 & 0.98 \\
\hline Stress (DASS-21) Time 1 & 8.02 & 4.67 & 0 & 18 & 0.38 & -0.49 \\
\hline Stress (DASS-21) Time 2 & 6.11 & 3.82 & 0 & 14 & 0.42 & -0.46 \\
\hline Stress (DASS-21) Time 3 & 7.02 & 4.69 & 0 & 17 & 0.47 & -0.64 \\
\hline \multicolumn{7}{|l|}{ Lockdown group } \\
\hline FFMQ-18 Time 1 & 59.65 & 7.77 & 47 & 75 & 0.38 & -0.73 \\
\hline FFMQ-18 Time 2 & 59.76 & 8.30 & 44 & 78 & 0.25 & -0.70 \\
\hline FFMQ-18 Time 3 & 60.27 & 8.25 & 46 & 78 & 0.09 & -0.55 \\
\hline SWLS Time 1 & 19.35 & 5.33 & 7 & 28 & -0.37 & -0.49 \\
\hline SWLS Time 2 & 19.73 & 5.77 & 6 & 27 & -0.57 & -0.65 \\
\hline SWLS Time 3 & 20.35 & 5.80 & 9 & 30 & -0.43 & -0.85 \\
\hline Depression (DASS-21) Time 1 & 5.68 & 4.39 & 0 & 19 & 1.19 & 1.51 \\
\hline Depression (DASS-21) Time 2 & 6.54 & 4.95 & 0 & 20 & 1.01 & 0.87 \\
\hline Depression (DASS-21) Time 3 & 5.49 & 4.10 & 0 & 16 & 0.79 & 0.07 \\
\hline Anxiety (DASS-21) Time 1 & 4.00 & 3.53 & 0 & 14 & 1.41 & 1.71 \\
\hline Anxiety (DASS-21) Time 2 & 4.11 & 3.66 & 0 & 13 & 1.11 & 0.21 \\
\hline Anxiety (DASS-21) Time 3 & 3.81 & 3.20 & 0 & 12 & 0.93 & 0.15 \\
\hline Stress (DASS-21) Time 1 & 6.65 & 3.33 & 0 & 12 & -0.07 & -1.05 \\
\hline Stress (DASS-21) Time 2 & 6.81 & 4.02 & 0 & 16 & 0.63 & -0.11 \\
\hline Stress (DASS-21) Time 3 & 6.92 & 4.00 & 0 & 16 & 0.27 & -0.40 \\
\hline
\end{tabular}




\section{Results}

The means, standard deviations, minimum and maximum values, and skewness and kurtosis values for all variables at each time point of the baseline group and the lockdown group are displayed in Table 2. The scales and important subscale scores implemented in this study were normally distributed, with skewness and kurtosis values ranging from -1.05 to 1.71 (West et al. (1995) stated that skewness and kurtosis values should fall between -2 and 2 for data to be considered normally distributed). There were no inequalities of variances across study variables between the baseline group and lockdown group. Mindfulness and life satisfaction levels at Time 1 were not significantly different between the baseline group and lockdown group, which permitted us to use these variables at Time 1 as additional predictors of distress outcomes in a mixed model.

For anxiety, a multi-level model showed a significant main effect of time $(F(1,79)=3.19, p=0.04), \eta 2=0.04)$, and interaction between group and time $(F(1,79)=5.44$, $p<0.01), \eta 2=0.07)$. The results indicated that anxiety levels changed in different ways over time depending on the group (baseline or lockdown). Post hoc tests show that for the baseline group, anxiety levels were significantly higher at Time 1 compared to Time 2 and Time $3(p=0.001)$, while no significant difference was observed for the lockdown group, (see Fig. 3), which was contrary to one of our original hypotheses. There were also significant main effects of mindfulness with a moderate effect size $(F(1,79)=5.42$, $p=0.023, \eta 2=0.07)$ and life satisfaction with a large effect size $(F(1,79)=11.83, p<0.001, \eta 2=0.13)$, indicating the protective roles of these variables against anxiety for both groups and over time.

A multi-level model was also applied for stress, which showed a significant main effect of time $(F(1,79)=5.92$, $p=0.003), \eta 2=0.07)$ and interaction between group and time $(F(1,79)=3.14, p=0.046), \eta 2=0.04)$. The results show stress levels changed in different ways over time depending on the group (baseline or lockdown). Post hoc tests indicated that for the baseline group, stress levels were significantly higher at Time 1 compared to Time $2(p=0.002)$ but not compared to Time 3 (see Fig. 4), which was contrary to one of our original hypotheses. The differences in the levels of stress between Time 2 and Time 3 were not significant for this group. For the lockdown group, anxiety levels did not change significantly over time. There was a significant and large main effect of mindfulness $(F(1,79)=15.64, p<0.001$, $\eta 2=0.17$ ), and a medium to large effect for satisfaction with life $(F(1,79)=10.31, p=0.002, \eta 2=0.12)$, indicating the protective roles of these variables against stress for both groups and over time.

There was no significant main effect of time and interaction between time and group observed for depression, indicating no significant differences between groups and over time. However, there was a significant and moderate main effect of mindfulness $(F(1,79)=6.15, p=0.015, \eta 2=0.07)$ and a large effect of life satisfaction $(F(1,79)=47.51$, $p<0.001, \eta 2=0.38$ ) on depression scores, signifying the protective nature of these variables against depression.
Fig. 3 Anxiety levels across the three time points of the baseline group $(n=44)$ and lockdown group $(n=37)$

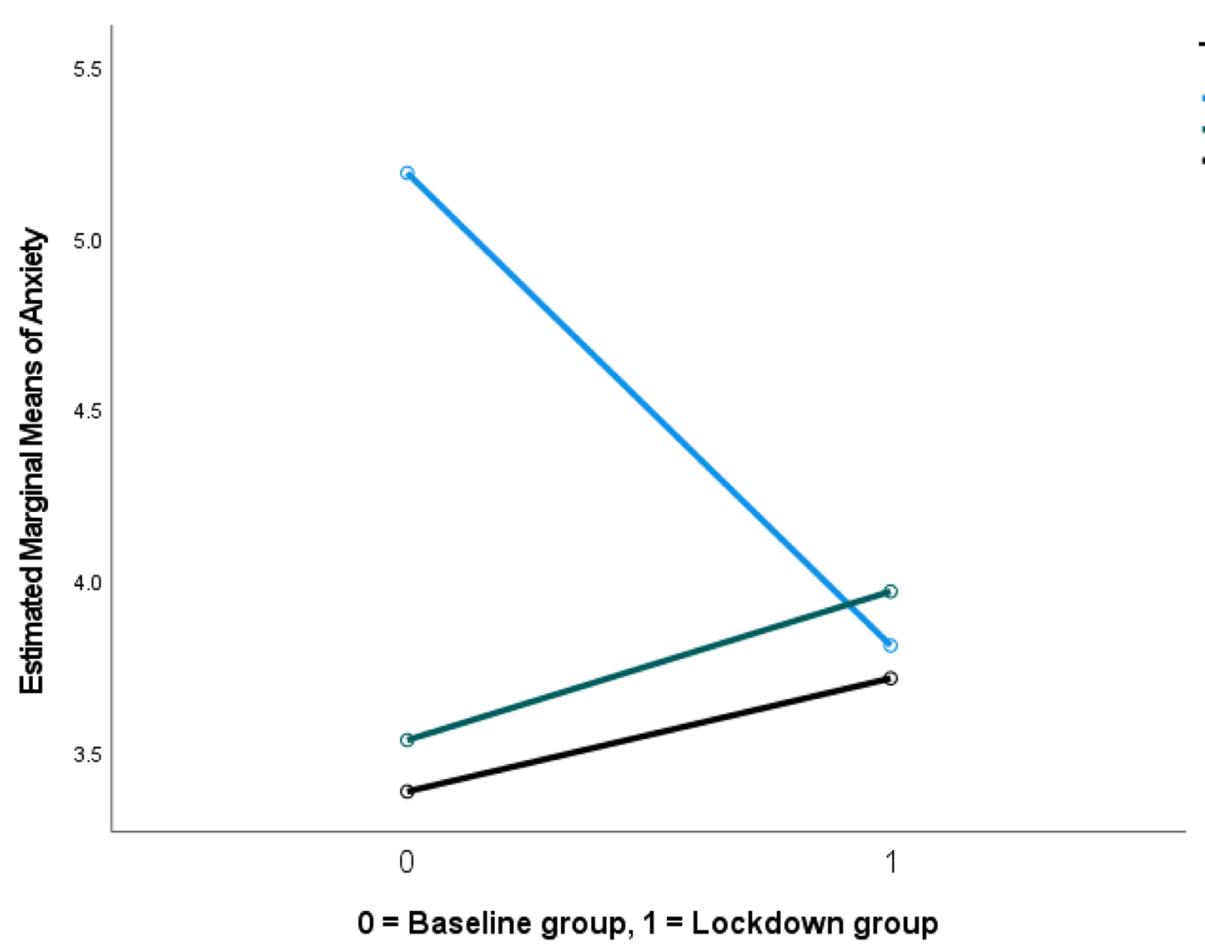

Time

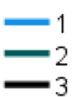


Fig. 4 Stress levels across the three time points of the baseline group $(n=44)$ and lockdown group $(n=37)$

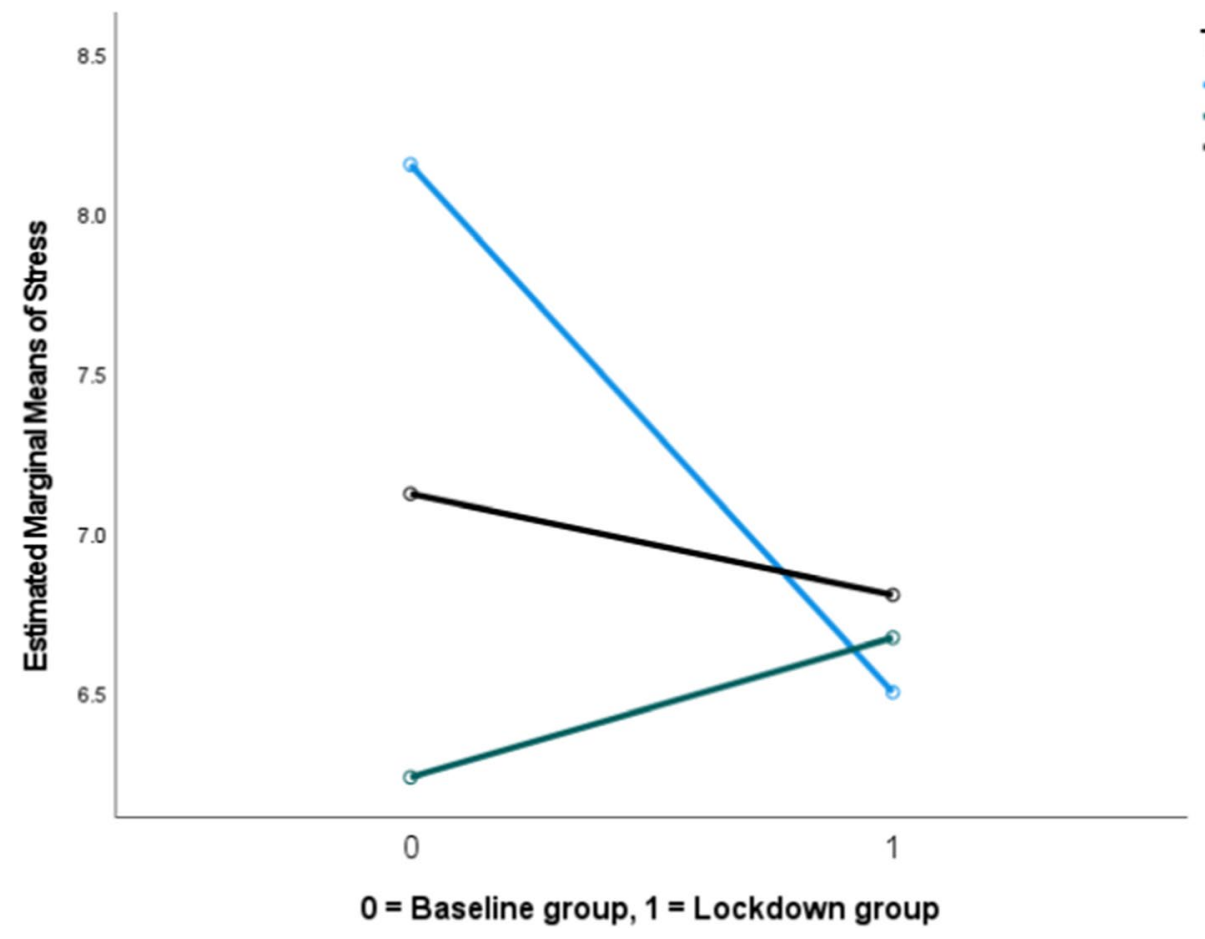

Regression analysis showed that when controlling for depression at Time $1\left(R^{2}=0.49, p<0.01\right)$ (the effect of the lockdown group was not significant), acting with awareness measured at Time 1 was the strongest significant inverse predictor of depression at Time 2 (standardized $\beta=-0.20$, $p=0.026$ ). No other mindfulness facets were significant predictors after controlling for the acting with awareness effect. Similarly, acting with awareness significantly and inversely predicted depression scores at Time 3 (standardized $\beta=-0.20, p=0.014)$ after accounting for depression at Time $1\left(R^{2}=0.55, p<0.01\right)$ while there was no significant effect of the lockdown group. A multiple linear regression model indicated that after controlling for anxiety at Time 1 $\left(R^{2}=0.45, p<0.01\right)$ and the significant effect of the lockdown group $\left(R^{2}=0.04, p=0.02\right)$, having a nonjudgmental attitude was the strongest predictor of anxiety at Time 2 , with a small to moderate effect size (standardized $\beta=-0.20$, $p=0.016$ ). After controlling for nonjudge, no other mindfulness facets were significant predictors of anxiety at Time 2 . When anxiety at Time 3 was used as the outcome variable, only anxiety at Time $1\left(R^{2}=0.45, p<0.01\right)$ and the lockdown group effects $\left(R^{2}=0.03, p=0.04\right)$ were significant predictors. Individual mindfulness facets measured at Time 1 were not significant predictors of stress at Time 2 or Time 3 after having controlled for stress at Time 1 and the effects of the lockdown group.

\section{Discussion}

The aim of the current study was to investigate anxiety, stress, and depression levels during lockdown in New Zealand using a quasi-experimental longitudinal research design controlling for baseline levels of mindfulness and satisfaction with life. The results showed that mindfulness and satisfaction with life at baseline significantly predicted lower levels of depression, anxiety, and stress during uncertain and emergency conditions, before and during lockdown, which aligned with our first hypothesis. Contrary to our second hypothesis, the results also indicate a significant reduction of anxiety and stress during lockdown compared to just prior to the lockdown baseline condition.

The findings suggest that individuals who have higher levels of mindfulness experience significantly less depression, anxiety, and stress over time, during both uncertain and emergency conditions such as during the threat of COVID19. The results are consistent with a study carried out in Italy showing that mindfulness was protective against psychological distress during the pandemic (Conversano et al., 2020). A study facilitated in Turkey (Saricali et al., 2020) demonstrated that lower levels of mindfulness were associated with greater levels of fear of COVID-19 using structural equation modeling with bootstrapping. As fear and/or worry are key components of anxiety, the findings of the current study are also consistent with the results of the study by Saricali et al. (2020) and demonstrate potential generalizability to other populations. 
The results of our study also suggest that people with higher levels of satisfaction with life experience significantly less depression, anxiety, and stress over time, during both uncertain and emergency conditions such as during the threat of COVID-19. The findings of the current study were consistent with research carried out in Poland during the onset of COVID-19 by Trzebiński et al. (2020), which showed that higher levels of life satisfaction were associated with lower levels of state anxiety, using correlational methods. The study by Trzebiński et al. further demonstrated that life satisfaction mediated the relationship between basic hope and both anxiety and COVID-19 stress, using mediational analyses. Similarly, a study facilitated with Italian participants during the pandemic by Gori et al. (2020) demonstrated the inverse relationship between life satisfaction and perceived stress using correlational analyses. The results of a study carried out with participants who were living in Canada in March and April 2020 (Best et al., 2020)—during which time strict social distancing was enforced by the Canadian Government-showed an inverse relationship between life satisfaction and depression, using correlational methods.

The current study adds weight to the findings of the relationships between mindfulness, satisfaction with life, and psychological distress described in other studies, because of its longitudinal scope. Correlational studies are valuable because they demonstrate important links between factors; however, it is not possible to detect the direction of the relationship as correlation does not determine causation. The point of difference of the current study is that data were collected across three time points, demonstrating mindfulness and satisfaction with life were predictive of lower psychological distress over time and in varying conditions.

The findings of the reduction in anxiety and stress after having gone into lockdown may be specific to the sample population as the New Zealand lockdown differed in many ways from those in other countries. Our findings are inconsistent with the results of the studies from the USA (Ettman et al., 2020), UK (Shevlin et al., 2020), and China (Wang et al., 2020) which were mentioned in the introduction section. Furthermore, a recent systematic review (Xiong et al., 2020) featuring studies from China, Denmark, Iran, Italy, Nepal, Spain, the USA, and Turkey indicated that anxiety, depression, and psychological distress were reported to be relatively higher during the COVID-19 pandemic, which is also inconsistent with the findings of the current study.

Another longitudinal study in New Zealand by Sibley et al. (2020) investigated the impact of COVID-19 on psychological outcomes with a large sample which was representative of the general population using a matchedsamples design. The results showed that the post-lockdown group reported somewhat higher levels of psychological distress compared to the pre-lockdown group. This finding is consistent with studies from other countries (see Xiong et al., 2020).

However, a noteworthy difference between the study by Sibley et al. (2020) and the current study is the time range during which data were collected. In the study by Sibley et al. (2020), participants in the pre-lockdown group completed the surveys from October 1 to December 31, 2019. The first cases of the virus in Wuhan, China, were not reported until December 1, and information about the disease was provided to the World Health Organization on December 31, 2019 (Strongman, 2020). The coronavirus was deemed low risk to New Zealanders in January 2020 by the Ministry of Health. Therefore, the study by Sibley et al. (2020) compares the psychological distress levels between individuals when the coronavirus was virtually unknown in New Zealand to those who were living through lockdown conditions due to COVID-19, whereas the current study compares depression, anxiety, and stress levels of participants just prior to the lockdown, when COVID-19 was known as a serious threat, and lockdown conditions. The results of the current study therefore indicate that the lockdown measures in New Zealand may have alleviated some of the stress and anxiety caused by the threat of COVID-19.

The results of the multiple linear regression we carried out to investigate the contributions of mindfulness facets to depression, anxiety, and stress demonstrated that acting with awareness assessed at Time 1 inversely predicted depression at Time 2 and Time 3, while other mindfulness facets were not significant predictors of depression after accounting for the acting with awareness effect. Unlike the results from the study by Medvedev et al. (2018a), which indicated having a nonjudgmental attitude was the strongest inverse predictor of depression, we found that the ability to act with awareness was the major inverse predictor of depression over time. This could be explained by the fact that the current study was conducted during the COVID-19 pandemic, including the lockdown condition. Therefore, the ability to act with awareness may be more effective in reducing depressive symptoms during uncertainty and emergency conditions. However, our results show that having a nonjudgmental attitude predicted lower levels of anxiety over time, which is fully consistent with the literature (Medvedev et al., 2018a) and emphasizes the important role this facet plays in protecting against anxiety in both normal and emergency conditions. Interestingly, after accounting for the variance explained by these major mindfulness predictors, other facets were not significant in predicting anxiety.

Preventative measures which increase mindfulness could help alleviate depression, anxiety, and stress during both uncertain and emergency conditions. Indeed, participants in studies who undertook mindfulness-based programs during the COVID-19 pandemic reported a reduction of depressive and anxiety symptoms. In a study in Italy (Matiz et al., 
2020), the depression and anxiety levels of female teacher participants significantly decreased after the completion of an 8-week mindfulness-oriented meditation program. A mindfulness-based intervention was implemented for cancer patients and their informal caregivers in California in a study by Kubo et al., (2019). The findings demonstrated that participants experienced less depression after having completed the program.

Preventative measures which help increase satisfaction with life could also help reduce depression, anxiety, and stress in uncertain and emergency conditions. A search in June 2021, using PsycInfo, Web of Science, and Scopus revealed that there were no peer-reviewed studies investigating the results of psychological treatments other than mindfulness-based interventions which aimed to enhance satisfaction with life solely during the COVID-19 pandemic. Interventions which have been successfully implemented at other times to help increase satisfaction with life have included strengths-based programs (Proctor et al., 2011), gratitude list-writing (Wood et al., 2010), hope enhancement strategies (Weis \& Speridakos, 2011), and the Best Possible Self intervention (Peters et al., 2013).

As described in the introduction, satisfaction with life is based on a cognitive, evaluative approach to one's life conditions (Diener et al., 1985) and therefore involves a type of self-judgment, which contrasts with the nonjudgmental facet of mindfulness. As outlined earlier, life satisfaction depends on evaluations based on various time perspectives, the present, as well as the past and future (Veenhoven, 2017), which further contrasts to the focus on the present moment in mindfulness. It follows that mindfulness could contribute to greater life satisfaction, because it engenders appreciating the present moment and learning to let go of judgments or taking a nonjudgmental stance towards one's own evaluations (Kabat-Zinn, 2001). Indeed, a mindfulnessbased intervention facilitated in Spain during the COVID-19 pandemic was shown to have significantly increased life satisfaction levels among a large sample of students (PizarroRuiz et al., 2021).

Interventions which help increase mindfulness and satisfaction with life may also improve immune responses during the COVID-19 pandemic. Research has shown that increased levels of stress are linked to decreased levels of immune functionality (Herbert \& Cohen, 1993). A meta-analysis demonstrated that mindfulness-based stress reduction leads to significant decreases in stress (Khoury et al., 2015), and other studies have shown the inverse relationship between stress and contentment or satisfaction with life (Cordaro et al., 2021; Schiffrin \& Nelson, 2010). Therefore, measures which help raise mindfulness and satisfaction with life could reduce stress, thereby enhancing immunity during uncertain and emergency conditions.

\section{Limitations and future research}

The current study was conducted in New Zealand, where the government had enforced measures which were different from those in other countries. The significant decreases in anxiety and stress experienced by the baseline group after having gone into the lockdown condition may not be generalizable to other populations, because conditions in New Zealand were (and continue to be) unique in many ways. Firstly, New Zealand is one of a few countries in the world to have a generally high standard of living. The information regarding the alert level system, requirements, and guidelines provided by the New Zealand Government throughout the pandemic was clear, concise, and comprehensive. The lockdown period in New Zealand was relatively short compared to those of other countries. Moreover, the requirements of citizens in New Zealand enforced by the government varied to those stipulated by governments of other regions around the world. A recent review (Brooks et al., 2020) demonstrated that inadequate supplies, inadequate information, and length of isolation impacted on distress levels during quarantine measures. Although the New Zealand-based sample limits the generalizability of some of the findings, the results also go some way to bear testimony to the effectiveness of the actions undertaken by the New Zealand Government during the COVID-19 crisis. However, the results which demonstrated that mindfulness and satisfaction with life protect against depression, anxiety, and stress in emergency and uncertain conditions may still be generalizable to other populations, given that these results applied to both groups and across all time points, and are consistent with the results of other studies.

The sample was made up of undergraduate university students, most of whom were young (between 18 and 29 years old) and female, which limits the generalizability of our findings to a wider population. The students were required to submit assessments with a maximum of a 1-week extension offered; therefore, the usual pressures associated with study were not especially alleviated. A transition from attending lectures and tutorials on campus to learning remotely, with lessons provided online was required of the students, which was not without its stressors. New Zealanders, including students, who lost their full-time or part-time jobs due to the effects of COVID-19 were able to access a relief payment ("Relief payments for people who lost jobs due to COVID19 announced," 2020). Given the unique set of stressors the students faced, it is difficult to determine whether the results of the decreases in anxiety and stress after having gone into lockdown would be generalizable to other populations, such as children and youth, retired persons, wage- and salaryearners, business-owners, or beneficiaries. 
In this study, we used the total mindfulness score to investigate the effects of mindfulness on psychological distress variables, due to using the short version of the FFMQ, in which reliability of individual subscales is less satisfactory than that of the total score. However, we conducted an exploratory regression analysis to investigate the contribution of individual mindfulness facets on depression, anxiety, and stress, although the results may be affected by lower reliability of individual facets. Future studies could use the full version of the FFMQ to allow for the investigation of the effects of mindfulness facets.

The findings of this study are limited as none of the items in the surveys asked the participants about their personal meditation experiences. Previous research demonstrates that individuals who meditate respond to FFMQ items differently to non-meditators (Van Dam et al., 2009). Responses to the observing facet in particular vary between meditators and non-meditators (Baer, 2011; Baer et al., 2006; Lilja et al., 2011). It is therefore difficult to make generalizable statements, especially in terms of individual facets, based on the results from data collected using the FFMQ when samples consist of individuals who probably vary substantially in relation to meditation practice.

Due to the inclusion of self-reported measures in the current study, a further limitation is common method bias. Spurious effects may occur when several constructs are measured by means of common methods such as multipleitem scales due to the measures themselves, instead of the constructs under investigation. For example, social desirability and item priming effects may influence the way in which participants respond to certain items, which are independent of the actual correlations between constructs under investigation (Podsakoff et al., 2003, 2012).

A limitation of quasi-experimental designs is the absence of randomization (Reichardt, 2009). In non-equivalent group designs such as the one implemented in this study, the participants of each group could differ systematically. Although it was demonstrated the groups do not significantly differ in terms of demographic variance, they could vary in other ways, which could have affected the results. However, in the estimation of the researchers, the differences in the levels of anxiety and stress between the first and second time points observed across the two groups were due to the lockdown condition, and not due to the characteristics of the participants of the specific groups. In addition, the sample size in the current study was relatively modest, which may limit the generalizability of our findings.

Further research is needed on the preventative effects of mindfulness interventions and treatments which help increase satisfaction with life on mental health outcomes such as depression, anxiety, and stress in the face of
COVID-19 with samples from a range of populations. Interventions to increase mindfulness and raise levels of satisfaction with life vary in both content and mode of delivery, and more studies are needed to determine which types are most effective during times of uncertainty and in emergency conditions. Furthermore, a preventative program designed to increase both mindfulness and satisfaction with life levels simultaneously could be created and tested to potentially buffer against adverse mental health outcomes during uncertain times and emergency conditions. The effects of mindfulness and satisfaction with life on immune responses need to be studied experimentally and developing a specific intervention which encompasses both these factors may prove worthwhile.

Further research on the psychological impact of the effects of COVID-19 with a range of samples and in various conditions is necessary to inform mental health practices around the world. As different populations face unique sets of problems in terms of number of active cases, healthcare access, standard of living, supplies, and access to information, a bespoke approach to mental health treatment may be indicated. The COVID-19 pandemic situation continues to develop rapidly; therefore, research must be ongoing. Longitudinal datasets on mental health outcomes with similar timings to those of the current study may exist and it would be beneficial for such data to be analyzed wherever possible. Further analysis of longitudinal data would allow for a more thorough understanding and comparison of critical time points at which the pandemic effects were affecting mental well-being. Longitudinal and quasi-experimental studies could inform preventative measures and mental health treatments in terms of timing and content.

Supplementary Information The online version contains supplementary material available at https://doi.org/10.1007/s12671-021-01731-4.

Acknowledgements We would like to thank all participants who were involved in this study. The data used in this study was from the Master of Social Science work of Jayne Hartstone at the University of Waikato.

Author Contribution JH designed the study, collected and analyzed the data, wrote the manuscript, and contributed to the critical revision and editing of the manuscript. OM contributed to the development of the study design, supervised the data analysis, and contributed to the critical revision of the manuscript. Both authors read and approved the final manuscript.

Funding No funds, grants, or other support was received.

Data Availability The data that support the findings of this study are available on request from the corresponding author. The data are not publicly available due to privacy or ethical restrictions. 


\section{Declarations}

Ethics approval All activities involving human participants were facilitated in alignment with the ethical standards of the Human Research Ethics Committee of the Division of Arts, Law, Social Science and Psychology, University of Waikato, New Zealand, and with the 1964 Helsinki declaration and its later amendments or comparable ethical standards.

Informed Consent All participants involved in the study gave informed consent online.

Conflict of Interest The authors declare that they have no conflict of interest.

\section{References}

American Psychological Association. (2020). Life Satisfaction. APA Dictionary of Psychology. https://dictionary.apa.org/life-satisfacti on

Antony, M. M., Bieling, P. J., Cox, B. J., Enns, M. W., \& Swinson, R. P. (1998). Psychometric properties of the 42-item and 21-item versions of the Depression Anxiety Stress Scales in clinical groups and a community sample. Psychological Assessment, 10(2), 176181. https://doi.org/10.1037/1040-3590.10.2.176

Auyeung, L., \& Mo, P. K. H. (2019). The efficacy and mechanism of online positive psychological intervention (PPI) on improving well-being among Chinese university students: A pilot study of the best possible self (BPS) intervention. Journal of Happiness Studies, 20, 2525-2550. https://doi.org/10.1007/s10902-018-0054-4

Baer, R. A. (2003). Mindfulness training as a clinical intervention: A conceptual and empirical review. Clinical Psychology: Science and Practice, 10(2), 125-143. https://doi.org/10.1093/clipsy. bpg015

Baer, R. A. (2011). Measuring mindfulness. Contemporary Buddhism, 12(1), 241-261. https://doi.org/10.1080/14639947.2011.564842

Baer, R. A., Smith, G. T., Hopkins, J., Krietemeyer, J., \& Toney, L. (2006). Using self-report assessment methods to explore facets of mindfulness. Assessment, 13(1), 27-45. https://doi.org/10.1177/ 1073191105283504

Beaumont, P. (2021). Decades of progress on extreme poverty now in reverse due to COVID. The Guardian. https://www.theguardian. com/global-development/2021/feb/03/decades-of-progress-onextreme-poverty-now-in-reverse-due-to-covid

Best, L. A., Law, M. A., Roach, S., \& Wilbiks, J. M. P. (2020). The psychological impact of COVID-19 in Canada: Effects of social isolation during the initial response. Canadian Psychology/psychologie Canadienne, 62(1), 143-154. https://doi.org/10.1037/cap0000254

Brooks, S. K., Webster, R. K., Smith, L. E., Woodland, L., Wessely, S., Greenberg, N., \& Rubin, G. J. (2020). The psychological impact of quarantine and how to reduce it: Rapid review of the evidence. The Lancet, 395, 912-920. https://doi.org/10.1016/ s0140-6736(20)30460-8

Brown, K. W., \& Ryan, R. M. (2003). The benefits of being present: mindfulness and its role in psychological well-being. Journal of Personality and Social Psychology, 84(4), 822-848. https://doi. org/10.1037/0022-3514.84.4.822

Chiesa, A., \& Serretti, A. (2009). Mindfulness-based stress reduction for stress management in healthy people: a review and meta-analysis. The Journal of Alternative and Complementary Medicine, 15(5), 593-600. https://doi.org/10.1089/acm.2008.0495

Conversano, C., Di Giuseppe, M., Miccoli, M., Ciacchini, R., Gemignani, A., \& Orrù, G. (2020). Mindfulness, age and gender as protective factors against psychological distress during COVID-19 pandemic. Frontiers in Psychology, 11, 1-9. https://doi.org/10. 3389/fpsyg.2020.01900

Cordaro, D. T., Bradley, C., Zhang, J. W., Zhu, F., \& Han, R. (2021). The development of the Positive Emotion Assessment of Contentment Experience (PEACE) scale. Journal of Happiness Studies, 22(4), 1769-1790. https://doi.org/10.1007/s10902-020-00295-9

Coronavirus: Lockdown eased in Moscow after nine weeks. (2020). BBC News. https://www.bbc.com/news/world-europe-52875568

Cummins, R. A., \& Nistico, H. (2002). Maintaining life satisfaction: the role of positive cognitive bias. Journal of Happiness Studies, 3, 37-69. https://doi.org/10.1023/A:1015678915305

Cunha, L. F., Pellanda, L. C., \& Reppold, C. T. (2019). Positive psychology and gratitude interventions: A randomized clinical trial. Frontiers in Psychology, 10, 1-9. https://doi.org/10.3389/fpsyg. 2019.00584

Diener, E., Emmons, R. A., Larsen, R. J., \& Griffin, S. (1985). The Satisfaction with Life Scale. Journal of Personality Assessment, 49(1), 71-75. https://doi.org/10.1207/s15327752jpa4901_13

Ettman, C. K., Abdalla, S. M., Cohen, G. H., Sampson, L., Vivier, P. M., \& Galea, S. (2020). Prevalence of depression symptoms in US adults before and during the Covid-19 pandemic. JAMA Network Open, 3(9), e2019686. https://doi.org/10.1001/jamanetworkopen. 2020.19686

Gallego, J., Aguilar-Parra, J. M., Cangas, A. J., Langer, Á. I., \& Mañas, I. (2014). Effect of a mindfulness program on stress, anxiety and depression in university students. Spanish Journal of Psychology, 17, 1-6. https://doi.org/10.1017/sjp.2014.102

Gori, A., Topino, E., \& Di Fabio, A. (2020). The protective role of life satisfaction, coping strategies and defense mechanisms on perceived stress due to COVID-19 emergency: A chained mediation model. PLoS ONE, 15(11), e242402. https://doi.org/10.1371/ journal.pone. 0242402

Gunia, A. (2020). Why New Zealand's coronavirus elimination strategy is unlikely to work in most other places. Time. https://time.com/ 5824042/new-zealand-coronavirus-elimination/

Headey, B., Kelley, J., \& Wearing, A. (1993). Dimensions of mental health: Life satisfaction, positive affect, anxiety and depression. Social Indicators Research, 29, 63-82. https://doi.org/10.1007/ BF01136197

Herbert, T. B., \& Cohen, S. (1993). Stress and immunity in humans: A meta-analytic review. Psychosomatic Medicine, 55(4), 364-379. https://doi.org/10.1097/00006842-199307000-00004

International Labour Organization. (2021). Slow jobs recovery and increased inequality risk long-term COVID-19 scarring. https:// www.ilo.org/global/about-the-ilo/newsroom/news/WCMS_ 794834/lang--en/index.htm

Jones, A. (2020). How did New Zealand become COVID-19 free? BBC News. https://www.bbc.com/news/world-asia-53274085

Joss, D., Khan, A., Lazar, S. W., \& Teicher, M. H. (2019). Effects of a mindfulness-based intervention on self-compassion and psychological health among young adults with a history of childhood maltreatment. Frontiers in Psychology, 10, 2373. https://doi.org/ 10.3389/fpsyg.2019.02373

Kabat-Zinn, J. (2001). Mindfulness meditation in everyday life. Piatkus Books.

Kabat-Zinn, J. (2015). Mindfulness. Mindfulness, 6, 1481-1483. https://doi.org/10.1007/s12671-015-0456-x

Khoury, B., Sharma, M., Rush, S. E., \& Fournier, C. (2015). Mindfulness-based stress reduction for healthy individuals: A metaanalysis. Journal of Psychosomatic Research, 78(6), 519-528. https://doi.org/10.1016/j.jpsychores.2015.03.009

Koydemir, S., \& Sun-Selışık, Z. E. (2016). Well-being on campus: Testing the effectiveness of an online strengths-based intervention for first year college students. British Journal of Guidance \& 
Counselling, 44(4), 434-446. https://doi.org/10.1080/03069885. 2015.1110562

Krägeloh, C. U., Henning, M. A., Medvedev, O. N., Feng, X. J., Moir, F., Billington, R., \& Siegert, R. J. (2019). Mindfulness-Based Intervention Research: Characteristics, Approaches, and Developments. Routledge. https://doi.org/10.4324/9781315545875

Kubo, A., Kurtovich, E., McGinnis, M., Aghaee, S., Altschuler, A., Quesenberry, C., Kolevska, T., \& Avins, A. L. (2019). A randomized controlled trial of mHealth mindfulness intervention for cancer patients and informal cancer caregivers: A feasibility study within an integrated health care delivery system. Integrative Cancer Therapies, 18, 1-13. https://doi.org/10.1177/15347 35419850634

Lilja, J. L., Frodi-Lundgren, A., Hanse, J. J., Josefsson, T., Lundh, L. G., Sköld, C., \& Broberg, A. G. (2011). Five Facets Mindfulness Questionnaire - reliability and factor structure: A Swedish version. Cognitive Behaviour Therapy, 40(4), 291-303. https:// doi.org/10.1080/16506073.2011.580367

Lovibond, S. H. \& Lovibond, P. F. (1993). Manual for the Depression Anxiety Stress Scales (DASS). Psychology Foundation Monograph.

Matiz, A., Fabbro, F., Paschetto, A., Cantone, D., Paolone, A. R., \& Crescentini, C. (2020). Positive impact of mindfulness meditation on mental health of female teachers during the COVID19 outbreak in Italy. International Journal of Environmental Research and Public Health, 17(18), 6450. https://doi.org/10. 3390/ijerph17186450

McMullan, L., Duncan, P., Blight, G., Gutiérrez, P., \& Hulley-Jones, F. (2021). COVID chaos: how the UK handled the coronavirus crisis. The Guardian. https://www.theguardian.com/world/ ng-interactive/2020/dec/16/covid-chaos-a-timeline-of-the-ukshandling-of-the-coronavirus-crisis

Medvedev, O. N., Cervin, M., Barcaccia, B., Siegert, R. J., Roemer, A., \& Krägeloh, C. U. (2020). Network analysis of mindfulness facets, affect, compassion, and distress. Mindfulness, 1-12. https://doi.org/10.1007/s12671-020-01555-8

Medvedev, O. N., Norden, P. A., Krägeloh, C. U., \& Siegert, R. J. (2018a). Investigating unique contributions of dispositional mindfulness facets to depression, anxiety, and stress in general and student populations. Mindfulness, 9, 1757-1767. https://doi. org/10.1007/s12671-018-0917-0

Medvedev, O. N., Titkova, E. A., Siegert, R. J., Hwang, Y. S., \& Krägeloh, C. U. (2018b). Evaluating short versions of the Five Facet Mindfulness Questionnaire using Rasch analysis. Mindfulness, 9, 1411-1422. https://doi.org/10.1007/s12671-017-0881-0

Nagy, L. M., \& Baer, R. A. (2017). Mindfulness: What should teachers of psychology know? Teaching of Psychology, 44(4), 353359. https://doi.org/10.1177/0098628317727913

Nielson, M. (2020). COVID-19 coronavirus: what will alert level 4 mean for New Zealand? New Zealand Herald. https://www. nzherald.co.nz/nz/covid-19-coronavirus-what-will-alert-level-4mean-for-new-zealand/7Z4NTSGEPQ6ZMZUU2H7QLR27IM/

Peters, M. L., Meevissen, Y. M. C., \& Hanssen, M. M. (2013). Specificity of the Best Possible Self intervention for increasing optimism: Comparison with a gratitude intervention. Terapia Psicológica, 31(1), 93-100. https://doi.org/10.4067/S0718-480820130001000 09

Pizarro-Ruiz, J. P., Ordóñez-Camblor, N., Del-Líbano, M., \& EscolarLLamazares, M. C. (2021). Influence on forgiveness, character strengths and satisfaction with life of a short mindfulness intervention via a Spanish smartphone application. International Journal of Environmental Research and Public Health, 18(2), 802. https://doi.org/10.3390/ijerph18020802

Podsakoff, P. M., MacKenzie, S. B., Lee, J.-Y., \& Podsakoff, N. P. (2003). Common method biases in behavioral research: A critical review of the literature and recommended remedies. Journal of Applied Psychology, 88(5), 879-903. https://doi.org/10.1037/ 0021-9010.88.5.879

Podsakoff, P. M., MacKenzie, S. B., \& Podsakoff, N. P. (2012). Sources of method bias in social science research and recommendations on how to control it. Annual Review of Psychology, 63, 539-569. https://doi.org/10.1146/annurev-psych-120710-100452

Proctor, C., Tsukayama, E., Wood, A. M., Maltby, J., Eades, J. F., \& Linley, P. A. (2011). Strengths gym: The impact of a character strengths-based intervention on the life satisfaction and wellbeing of adolescents. The Journal of Positive Psychology, 6(5), 377-388. https://doi.org/10.1080/17439760.2011.594079

Reichardt, C. S. (2009). Quasi-experimental design. In R. E. Millsap \& A. Maydeu-Olivares (Eds.), The SAGE handbook of quantitative methods in psychology (pp. 46-71). Sage Publications Ltd. https:// doi.org/10.4135/9780857020994.n3

Relief payments for people who lost jobs due to COVID-19 announced. (2020). RNZ. https://www.rnz.co.nz/news/political/417450/reliefpayments-for-people-who-lost-jobs-due-to-covid-19-announced

Roemer, A., Sutton, A., Grimm, C., \& Medvedev, O. N. (2021a). Differential contribution of the five facets of mindfulness to wellbeing and psychological distress. Mindfulness, 12, 693-700. https://doi.org/10.1007/s12671-020-01535-y

Roemer, A., Sutton, A., Grimm, C., \& Medvedev, O. N. (2021b). Effectiveness of a low-dose mindfulness-based intervention for alleviating distress in young unemployed adults. Stress and Health, 37(2), 320-328. https://doi.org/10.1002/smi.2997

Saricali, M., Satici, S. A., Satici, B., Gocet-Tekin, E., \& Griffiths, M. D. (2020). Fear of COVID-19, mindfulness, humor, and hopelessness: a multiple mediation analysis. International Journal of Mental Health and Addiction, 1-14. https://doi.org/10.1007/ s11469-020-00419-5

Schiffrin, H. H., \& Nelson, S. K. (2010). Stressed and happy? Investigating the relationship between happiness and perceived stress. Journal of Happiness Studies, 11(1), 33-39. https://doi.org/10. 1007/s10902-008-9104-7

Shevlin, M., McBride, O., Murphy, J., Miller, J. G., Hartman, T. K., Levita, L., Mason, L., Martinez, A. P., McKay, R., Stocks, T. V., Bennett, K. M., Hyland, P., ThanosKaratzias, T., \& Bentall, R. P. (2020). Anxiety, depression, traumatic stress and COVID-19-related anxiety in the UK general population during the COVID-19 pandemic. Bjpsych Open, 6(6), E125. https://doi.org/10.1192/bjo. 2020.109

Sibley, C. G., Greaves, L. M., Satherley, N., Wilson, M. S., Overall, N. C., Lee, C. H. J., Milojev, P., Bulbulia, J., Osborne, D., Milfont, T. L., Houkamau, C. A., Duck, I. M., Vickers-Jones, R., \& Barlow, F. K. (2020). Effects of the COVID-19 pandemic and nationwide lockdown on trust, attitudes toward government, and well-being. American Psychologist, 75(5), 618-630. https://doi.org/10.1037/ amp0000662

Strauss, C., Cavanagh, K., Oliver, A., \& Pettman, D. (2014). Mindfulness-based interventions for people diagnosed with a current episode of an anxiety or depressive disorder: A meta-analysis of randomised controlled trials. PLoS ONE, 9(4), e96110. https:// doi.org/10.1371/journal.pone.0096110

Strongman, S. (2020). COVID-19 pandemic timeline: How the coronavirus started, spread and stalled life in New Zealand. RNZ. https://shorthand.radionz.co.nz/coronavirus-timeline/

The Atlantic Monthly Group (2021). The data. The COVID Tracking Project. https://covidtracking.com/data

The World Bank. (2021). Food Security and COVID-19. https:// www.worldbank.org/en/topic/agriculture/brief/food-secur ity-and-covid-19

Truong, Q. C., Krägeloh, C. U., Siegert, R. J., Landon, J., \& Medvedev, O. N. (2020). Applying generalizability theory to differentiate between trait and state in the Five Facet Mindfulness 
Questionnaire (FFMQ). Mindfulness, 11, 953-963. https://doi.org/ 10.1007/s12671-020-01324-7

Trzebiński, J., Cabański, M., \& Czarnecka, J. Z. (2020). Reaction to the COVID-19 pandemic: The influence of meaning in life, life satisfaction, and assumptions on world orderliness and positivity. Journal of Loss and Trauma, 25(6-7), 544-557. https://doi.org/ $10.1080 / 15325024.2020 .1765098$

Twenge, J. M., \& Joiner, T. E. (2020). US Census Bureau-assessed prevalence of anxiety and depressive symptoms in 2019 and during the 2020 COVID-19 pandemic. Depression and Anxiety, 37(10), 954-956. https://doi.org/10.1002/da.23077

van Beuningen, J. (2012). The Satisfaction with Life Scale: Examining construct validity. Statistics Netherlands.

Van Dam, N. T., Earleywine, M., \& Danoff-Burg, S. (2009). Differential item function across meditators and non-meditators on the Five Facet Mindfulness Questionnaire. Personality and Individual Differences, 47(5), 516-521. https://doi.org/10.1016/j.paid.2009. 05.005

Veenhoven, R. (2017). Happiness research: Past and future. Senshu Social Well-Being Review, 2017(4), 65-73.

Vøllestad, J., Nielsen, M. B., \& Nielsen, G. H. (2012). Mindfulnessand acceptance-based interventions for anxiety disorders: A systematic review and meta-analysis. British Journal of Clinical Psychology, 51(3), 239-260. https://doi.org/10.1111/j.2044-8260. 2011.02024.x

Wang, C., Pan, R., Wan, X., Tan, Y., Xu, L., McIntyre, R. S., Choo, F. N., Tran, B., Ho, R., Sharma, V. K., \& Ho, C. (2020). A longitudinal study on the mental health of general population during the COVID-19 epidemic in China. Brain, Behavior, and Immunity, 87, 40-48. https://doi.org/10.1016/j.bbi.2020.04.028
Weis, R., \& Speridakos, E. C. (2011). A meta-analysis of hope enhancement strategies in clinical and community settings. Psychology of Well-Being: Theory, Research and Practice, 1(5), 1-16. https://doi.org/10.1186/2211-1522-1-5

West, S. G., Finch, J. F., \& Curran, P. J. (1995). Structural equation models with nonnormal variables: problems and remedies. In R. H. Hoyle (Ed.), Structural equation modeling: Concepts, issues, and applications (pp. 56-75). Sage Publications Inc.

Wood, A. M., Froh, J. J., \& Geraghty, A. W. A. (2010). Gratitude and well-being: A review and theoretical integration. Clinical Psychology Review, 30(7), 890-905. https://doi.org/10.1016/j.cpr. 2010.03.005

World Health Organization. (2020). COVID-19 disrupting mental health services in most countries, WHO survey. https://www.who. int/news/item/05-10-2020-covid-19-disrupting-mental-healthservices-in-most-countries-who-survey

Worldometer. (2021). COVID-19 coronavirus pandemic. Retrieved August 15, 2021, from https://www.worldometers.info/coron avirus/

Xiong, J., Lipsitz, O., Nasri, F., Lui, L. M. W., Gill, H., Phan, L., Chen-Li, D., Iacobucci, M., Ho, R., Majeed, A., \& McIntyre, R. S. (2020). Impact of COVID-19 pandemic on mental health in the general population: A systematic review. Journal of Affective Disorders, 277, 55-64. https://doi.org/10.1016/j.jad.2020.08.001

Publisher's Note Springer Nature remains neutral with regard to jurisdictional claims in published maps and institutional affiliations. 